\title{
Diabetes Mellitus: Aptitud clínica del médico de atención primaria
}

\section{Diabetes Mellitus: Clinical aptitude of the doctor of primary care.}

Víctor Manuel Gómez-López*, Martha Elena García-Ruiz**, Carlos Barrientos- Guerrero ***.

Objetivo: Comparar la aptitud clínica del médico de las Unidades de Medicina Familiar, en la atención de la diabetes mellitus.

Material y métodos: Diseño transversal y comparativo. Se aplicó un instrumento de evaluación validado previamente por un grupo de expertos, a 78 médicos familiares que se desempeñan en el primer nivel de atención. Dentro de los indicadores explorados con el instrumento de evaluación se incluyen:

I Reconocimiento de factores de riesgo,

II Reconocimiento de signos y síntomas, III Utilización e interpretación de recursos de laboratorio y gabinete, IV Integración diagnóstica, $\mathrm{V}$ Utilización de medidas terapéuticas y VI Medidas de seguimiento.

Para el análisis estadístico, se utilizó la prueba de Kruskall-Wallis y la Ji cuadrada con un nivel de significancia de 0.05

Resultados: El puntaje que correspondió a lo explicable por efectos del azar fue $<24$ en la calificación global.

De acuerdo a la escala utilizada, el 64 \% (IC $95 \%$ de 53 a $70 \%$ ) de los resultados de las calificaciones, se ubicó en la escala baja (49-73) En general no hubo diferencias significativas en los resultados de la aptitud clínica por indicador y grado académico.
Correspondencia:

Dr. Víctor Manuel Gómez López

Domicilio: Juárez 1203 Sur,

Colonia Obrera Cd. Madero, Tamaulipas, México.

Teléfono: 01-833- 210-06-27 (Casa)

e-mail: vmgomezl@yahoo.com
De acuerdo a los objetivos del estudio, la asociación entre grado académico y aptitud clínica utilizando la Ji cuadrada fue de 1.331 ( $p=N S$ ).

Conclusiones: No se encontró diferencia estadísticamente significativa en la aptitud clínica entre los médicos generales, especialistas en medicina familiar y especialistas certificados.

Palabras clave: Aptitud clínica, diabetes mellitus, primer nivel de atención.

Objective: to compare the clinic aptitude of the doctor in the unities of familiar medicine in the care of the diabetes mellitus.

Material and Methods: Transversal and comparative pattern. A evaluation previously validated by a group of experts was apply to $\mathbf{7 8}$ specialist in familiar medicine who redeem in the first level of attention. The indicators explored in the evaluation are. I recognition of cause of risk. II recognition of signs and symptoms. III utilization and interpretation of laboratory studies and consultation studies. IV diagnostic integration. $V$ utilization of terapeutic measures and VI following measures.

For the statistical analysis, it was used the Kruskall-Wallis and chi-square with a level of important of 0.05

Results: the points to the explainable for effects of chance were of $<24$ in global grade. According to the scale used, the $64 \%$ (IC $95 \%$ de 53 a $70 \%$ ) of the results in the grades was situated in the low scale (49-73). In general it didn't appear significative differences in the results of clinic aptitude by indicator and academic degree. According to objetives 
of the study, the association between academic degree and the clinic aptitude using the chi-square was $1.331(p=N S)$

Conclusions: We didn't find statistically significative difference in the clinic aptitude among the general doctors, specialists in familiar medicine and certificated specialist.

Key words: Clinic aptitude, diabetes mellitus first level of attention.

\section{INTRODUCCIÓN}

La visión tradicional del médico, lo identifica como un clínico, es decir, como un profesional que atiende pacientes. La clínica se puede conceptualizar como la parte práctica de la medicina, la aplicación del conocimiento médico a la solución de los problemas de los pacientes, la habilidad para atender a los pacientes, o como una competencia o un conjunto de competencias ${ }^{1}$.

En su concepción más simple, evaluar es comparar una medida con un estándar o patrón y emitir un juicio valorativo basado en esa comparación. La evaluación ha sido y continuará siendo un gran desafío para los educadores en virtud de la diversidad de modificaciones que sufren los educandos como resultado de su exposición a numerosas experiencias de aprendizaje ${ }^{2}$.

En México, Viniegra y colaboradores ${ }^{3-5}$ han desarrollado y perfeccionado instrumentos orientados a la medición de competencias clínicas, profundizando en la utilidad de exámenes escritos para medir capacidad clínicas complejas.

La competencia clínica que constituye una parte trascendental del desempeño de los profesionales clínicos también es entendida como una determinante de este; se expresa en el grado de pericia de los médicos para afrontar con éxito problemas clínicos con base en la reflexión y la crítica aplicadas a distintas situaciones clínicas; es decir constituye el conjunto de capacidades involucradas con la detección y resolución de problemas clínicos suscitados en la práctica clínica cotidiana, en sus aspectos de prevención, diagnóstico, tratamiento y seguimiento ${ }^{6}$.

Para evaluar la competencia clínica se requiere de una estrategia de indagación estructurada en base a lo que llamamos problematización de las situaciones clínicas descritas (casos clínicos reales); esto significa que el respondiente de los instrumentos utilizados debe poner en juego su propio criterio al reconocer con mayor o menor claridad las particularidades de cada situación clínica que enfrenta, identificar las alternativas que se proponen con fines de diagnóstico tratamiento o prevención y diferenciar las que a su juicio resultan apropiadas, útiles, oportunas o beneficiosas, de las inapropiadas, inútiles, extemporáneas, inconvenientes o perjudiciales. Es en este sentido que los instrumentos de medición de la competencia clínica nos permiten reconstruir la experiencia de aprendizaje de quien responde ${ }^{7}$.

Reconocemos que la aptitud clínica debe explorarse en el sitio donde se desarrolla la actividad clínica. Sin embargo los instrumentos aquí utilizados están orientados a medir la aptitud clínica y constituyen una alternativa adecuada y factible para los propósitos que aquí se persiguen. Los instrumentos utilizados por nosotros fueron validados y sobre todo tienen pertinencia en cuanto a los indicadores que exploran como son reconocimiento de factores de riesgo, reconocimiento de signos y síntomas, integración diagnóstica, uso de estudios de laboratorio y recursos terapéuticos, comisión u omisión de conductas iatrogénicas perjudiciales ${ }^{8,9}$.

Ya se ha investigado la competencia y desempeño clínico de médicos del primer nivel de atención en el diagnóstico y manejo de pacientes con diabetes mellitus tipo $2^{10}$, por lo que el propósito principal en la presente investigación es comparar la aptitud clínica del médico en la atención de la diabetes mellitus, según grado académico alcanzado.

\section{MATERIAL Y MÉTODOS}

Para efectos de este trabajo se entiende por aptitud clínica la competencia del médico para identificar y resolver apropiadamente las situaciones clínicas problema, representadas por casos clínicos reales de pacientes con diabetes mellitus, contenidos en un instrumento de medición construido con este fin. Cuando la calificación obtenida estuvo ubicada en el rango de competencia alta (99-123 puntos), se consideró que el médico es apto para la atención de los pacientes con diabetes mellitus.

Para llevar a cabo este estudio se construyó un instrumento (ver anexo 1) con cuatro casos clínicos reales, condensados, seleccionados de la consulta externa de la unidad de atención primaria, más grande del estado de Tamaulipas, México, el cual fue 


\begin{tabular}{|c|c|c|c|c|}
\hline Escala de $\mathrm{AC}^{*}$ & $\begin{array}{l}\text { M. G. } \\
n=23\end{array}$ & $\begin{array}{r}\text { Esp. en M.F } \\
n=22\end{array}$ & $\begin{array}{l}\text { Esp. en M.F. certificado } \\
\qquad n=33\end{array}$ & $\begin{array}{l}\text { Total } \\
\mathrm{n}=78\end{array}$ \\
\hline Muy alta(124-148) & 0 & 0 & 0 & 0 \\
\hline Alta(99-123) & 0 & 0 & 0 & 0 \\
\hline Media(74-98) & 5 & 4 & 10 & 19 \\
\hline $\operatorname{Baja}(49-73)$ & 15 & 15 & 20 & 50 \\
\hline Muy baja (24-48) & 3 & 3 & 3 & 9 \\
\hline Explicable por azar & 0 & 0 & 0 & 78 \\
\hline $\begin{array}{l}\text { * Aptitud clínica. } \\
\text { MG: Médico general } \\
\text { MF: Medicina familiar }\end{array}$ & & & & \\
\hline
\end{tabular}

aplicado a 78 médicos que laboran como médicos familiares en cuatro unidades de medicina familiar de una institución de asistencia pública del mismo estado, de los cuales 23 correspondieron a médicos generales, quienes son médicos que solo cursaron la carrera de medicina, 22 a especialistas en medicina familiar y 33 a especialistas en medicina familiar certificados. La Certificación para ejercer la especialidad de medicina familiar la otorga el Consejo Mexicano de Certificación en Medicina Familiar A.C. El contenido del instrumento aplicado correspondió a aspectos que el médico debe realizar en el primer nivel de atención. Se exploraron indicadores que se relacionan con los niveles de atención del proceso salud enfermedad tales como; I Reconocimiento de factores de riesgo, II Reconocimiento de signos y síntomas, III Utilización e interpretación de recursos de laboratorio y gabinete, IV Integración diagnóstica, V Utilización de medidas terapéuticas y VI Medidas de seguimiento. El cuestionario incluyó opciones de respuestas de verdadero, falso y no sé, del cual una respuesta correcta suma un punto, una incorrecta resta un punto y la respuesta no sé ni sumaba ni restaba puntos. La calificación se obtuvo al restar el número de respuestas incorrectas al número de respuestas correctas. Inicialmente el instrumento diseñado estuvo constituido por 180 enunciados, el cual después de someterlo a dos rondas con expertos (Un Endocrinólogo, un Nefrólogo, dos Internis-tas, un Internista adscrito al servicio de Urgencias y un Médico Familiar con experiencia en la atención de la diabetes mellitus) se llegó por consenso a la descripción de los casos clínicos así como a 148 enunciados, de los cuales 78 correspondieron con respuesta correcta verdadero y 70 con respuesta correcta falso. El número de aseveraciones para cada uno de los indicadores del I al VI que fueron evaluados fue de 19, 10, 20, 37, 44 y 18 respectivamente.

Para el análisis de la aptitud clínica se estimó la distribución de los resultados por efecto del azar ${ }^{11} \mathrm{y}$ a partir de estos se construyó una escala de competencia de 24 a 148 puntos. Se utilizó la prueba de Kruskal Wallis para estimar las diferencias entre los tres grados académicos comparados ${ }^{12}$. Para estimar la asociación entre el grado académico y aptitud clínica, se utilizó la Ji cuadrada con un nivel de significancia de 0.05

\section{RESULTADOS}

La consistencia interna del instrumento de medición utilizado en este estudio se calculó mediante la prueba de Kuder-Richardson que arrojó un coeficiente de 0.84

En la tabla 1 se observa la distribución de los médicos de acuerdo a su calificación, en donde se aprecia que el $64 \%$ (IC $95 \%$ de 53 a $70 \%$ ) de ellos obtuvieron un resultado bajo, de acuerdo a la escala utilizada. El puntaje que correspondió a lo explicable por efectos del azar fue $<24$ en la calificación global.

En la tabla 2 se anotan las calificaciones de la aptitud clínica (AC) en función de los indicadores evaluados. La calificación global de cada grupo estuvo constituida por la suma del puntaje del total de indicadores que constituyeron el instrumento de evaluación utilizado. En general no hubo diferencias significativas en los resultados de la aptitud clínica por indicador y grado académico.

Por último, de acuerdo a los objetivos del estudio, la asociación entre grado académico y aptitud clínica utilizando la Ji cuadrada fue de 1.331 ( $\mathrm{p}=\mathrm{NS}$ ). 


\begin{tabular}{lrrrrr}
\hline \multicolumn{2}{l}{ Tabla 2: Aptitud Clínica por indicador y grado académico } & & & \\
\hline Indicador & \multicolumn{1}{c}{ Enunciados } & $\begin{array}{r}\text { M.G. } \\
\mathrm{n}=23\end{array}$ & $\begin{array}{r}\text { M.F } \\
\mathrm{n}=22\end{array}$ & $\begin{array}{r}\text { M.F.C } \\
\mathrm{n}=33\end{array}$ & $\mathrm{p}^{*}$ \\
\hline Resultado global & 148 & 59 & 59 & 66 & $\mathrm{~ns}$ \\
Reconocimiento de factores de riesgo & 19 & 14 & 14 & 14 & $\mathrm{~ns}$ \\
Reconocimiento de signos y sintomas & 10 & 6 & 7 & 6 & $\mathrm{~ns}$ \\
Utiliz. e Inter. de rec. de lab. y gabinete & 20 & 16 & 15 & 28 & $\mathrm{~ns}$ \\
Integración diagnostica & 37 & 27 & 27 & 32 & $\mathrm{~ns}$ \\
Medidas terapéuticas & 44 & 31 & 31 & 9 & $\mathrm{~ns}$ \\
Medidas de seguimiento & 18 & 10 & 9 & & \\
\hline
\end{tabular}

*p prueba de Kruskall - Wallis

\section{DISCUSIÓN}

En el presente estudio se comparó la aptitud clínica en diabetes mellitus que muestran los médicos de la atención primaria.

La exploración de la aptitud clínica, se basó en la idea de que es la reflexión sobre la experiencia clínica la que puede hacer la diferencia en cuanto al grado de utilización de dicha aptitud y no solamente el grado académico alcanzado.

En el análisis global de los resultados de la aptitud clínica, no se encontró diferencias significativas en la comparación de los tres grupos. Se esperaría que el médico familiar certificado por el Consejo correspondiente, mostrara mayor aptitud partiendo del supuesto de que ha estado expuesto a un mayor grado de formación académica. Sin embargo estos hallazgos pueden explicarse por el hecho de que la formación de nuestros médicos, está basada en el recuerdo, lo que evidentemente dificulta la capacidad para la reflexión ${ }^{13}$ ante situaciones clínicas problemáticas a las que se enfrenta cotidianamente, ya que su quehacer es rutinario sin analizar al paciente como individuo.

Es preciso reconocer sin embargo, que aunque la aptitud clínica aquí identificada se ubica en el rango de baja (49-73) en un porcentaje del $64 \%$, es superior a la obtenida por otros autores ${ }^{14}$.

Evidentemente que los casos clínicos no agotaron la diversidad de aquellos que acuden a la consulta; más bien representan a los que más comúnmente se atienden en unidades de primer contacto. Al ser extraídos de la consulta de ese tipo de unidades, el médico se enfrenta desde el punto de vista teórico a su solución y deberá considerar todos los factores propios de ese caso, desde la edad, etapa del curso clínico del padecimiento, patología concomitante, etc. para tomar decisiones.

Dado que la competencia clínica es una actividad compleja que comprende un conjunto de atributos multidimensionales y no solo un aspecto unidimensional ${ }^{15}$, los resultados encontrados tienen implicaciones educativas y operativas, puesto que manifiestan la necesidad de modificar las estrategias educativas utilizadas en la educación médica continua hacia otras tendencias donde se desarrolle la capacidad de la reflexión (crítica) de manera preponderante y además de supervisión del quehacer médico cotidiano ${ }^{16}$.

\section{BIBLIOGRAFÍA}

1. Laredo-Sánchez F, Lifshitz A. Introducción al estudio de la medicina clínica. Un nuevo enfoque. Editorial Prado. México. 2001

2. Viniegra-VL, Jiménez JL, Pérez-Padilla JR. El desafío de la evaluación de la competencia clínica. Rev Invest Clin. 1991; 43(1): 87-95

3. Viniegra L, Lisker R. Utilidad de los exámenes de selección múltiple en la evaluación del aprendizaje ocurrido durante el adiestramiento clínico intensivo. Rev Invest Clin 1979; 31: 407-12

4. Viniegra L, Ponce de León S, Lisker R. Efectos de la práctica clínica sobre los resultados de los exámenes de opción múltiple. Rev Invest Clin 1981; 33: 313-7

5. Viniegra L, Montes J, Sifuentes J, Uscanga L. Comparación de la utilidad de dos tipos de exámenes teóricos para evaluar el aprendizaje clínico. Rev Invest Clin 1982; 34: 73-8

6. García HA, Viniegra VL. Competencia clínica del médico familiar en hipertensión arterial sistémica. 1999; 51(2): 93-98.

7. Viniegra L Evaluación de la competencia clínica: describir o reconstruir. Rev Invest Clin 2000; 52(2): 109-110

8. Rivera ID, Aguilar ME, Viniegra VL. Evaluación de la aptitud 
clínica de médicos residentes de medicina física y rehabilitación. Rev Invest Clin 1998; 50(4): 341-6

9. Viniegra L, Jiménez JL. Nuevas aproximaciones a la medición de la competencia clínica. Rev Invest Clin 1992; 44(2): 269-75

10. Sabido SC, Viniegra VL. Competencia y desempeño clínicos en diabetes. Rev Invest Clin 1998; 50(3): 211-6

11. Pérez-Padilla JR, Viniegra-Velázquez L .Método para calcular la distribución de las calificaciones esperadas por azar en un examen del tipo falso, verdadero y no sé. Rev Invest Clin 1989; 41: 375-379

12. Dawson BS, Trapp RG. Bioestadística médica. México: Manual Moderno; 1997: 263
13. Viniegra VL. Hacia otra concepción del currículo. México. IMSS. 1999. pp 13-23

14. Trujillo-Galván FD, González- Cobos R, Munguía-Miranda C. Competencia clínica en diabetes mellitus. Rev Med IMSS 2002; 40(6): 467-472

15. Norman GR. Defining competence: a methodological review. En Neufeld VR, Norman GR. Editors Assessing Clinical Competente. New York. Springer Publishing Company; 1985 pp.15-37

16. Viniegra VL. La crítica: aptitud olvidada por la educación. México. IMSS. 2000. pp 3-4

Anexo 1 Ejemplo del instrumento aplicado para evaluar la aptitud clínica en diabetes mellitus.

\section{INSTRUCCIONES PARA RESPONDER CASOS CLINICOS.}

A continuación encontrará varios casos clínicos resumidos, cada uno de ellos se acompaña de un número de aseveraciones. Cada una de estas afirmaciones va seguida de enunciados referentes al caso que pueden ser falsas (F) o verdaderas (V) .

Lea cuidadosamente cada caso que cuenta con la información necesaria para responder cada enunciado. Para expresar sus respuestas: V, F, NS tome en cuenta exclusivamente lo que está descrito en cada uno de los casos. Utilice la línea que aparece a la izquierda de cada enunciado para anotar sus respuestas: " $\mathrm{F}$ " si considera que el enunciado es falso, " $\mathrm{V}$ " si considera que es cierto y NS en caso de que ignore o no pueda decidir si el enunciado es verdadero o falso. Tome en cuenta que: una respuesta correcta le suma un punto, una respuesta incorrecta le resta un punto, una respuesta no se, no le suma ni le resta puntos y un enunciado no respondido le resta un punto.

\section{TERMINOS EMPLEADOS EN ESTE INSTRUMENTO DE EVALUACION}

FACTOR DE RIESGO: Se refiere a la existencia demostrada de antecedentes o condiciones actuales del paciente, que sin formar parte de un padecimiento, hacen más probable su aparición o que este se agrave COMPATIBLE: Se refiere al hecho de que los elementos de un caso clínico concuerdan con la entidad nosológica propuesta como diagnóstico. A FAVOR: Presencia de un dato clínico, de laboratorio o gabinete que forma parte y es base importante en el diagnóstico de la enfermedad en cuestión.

UTIL: Hace referencia a ciertos procedimientos o medidas de diagnóstico que presentan claramente más beneficios (precisión diagnóstica) que perjuicios (molestias innecesarias) en la situación clínica descrita.

APROPIADO: Hace referencia a ciertas medidas o recomendaciones terapéuticas, preventivas o de seguimiento que son más efectivas en la situación clínica descrita, con escasos efectos indeseables y claros beneficios inmediatos potenciales

SEGUIMIENTO: Hace referencia a ciertos procedimientos o medidas pertinentes para la vigilancia y control del paciente descrito a corto, mediano y largo plazo.

FACTOR PRONOSTICO: Son signos o síntomas clínicos o paraclínicos que forman parte de un padecimiento que indican una evolución favorable o desfavorable.

ASOCIADO: Se refiere a la coexistencia demostrada de dos o más hechos o fenómenos ( signos, síntomas, modificaciones ) sin afirmar o negar relaciones de causa- efecto.

CARACTERISTICO: Presencia de un hecho o fenómeno ampliamente demostrado de manera tal que su ocurrencia debe hacer pensar en dicha asociación.

Femenina de 63 años de edad. Ocupación labores del hogar. Escolaridad segundo de primaria. G: V P: V. Padre diabético, falleció. Una hermana con diabetes mellitus. Tabaquismo negativo.
Cursa con diabetes mellitus desde hace 30 años. Hipertensa de 10 años de evolución. Además presenta artralgias de predominio en rodillas. Exploración física: Peso: 57 Kilogramos. Talla: 1.49 cm TA: 130/80 mm Hg. Consciente, bien orientada en las tres esferas, con buena coloración de tegumentos y conjuntivas. Rs. Cs. normales sin fenómenos agregados. Campos pulmonares con buena ventilación sin fenómenos agregados. Extremidades con pulsos presentes y normales para su edad. Desde hace 2 años ha acudido en 5 ocasiones con el médico familiar. En la última visita se revisan los resultados de los siguientes exámenes de laboratorio reportándose lo siguiente: En el examen general de orina Ph: 5 Densidad de 1000 , cetonas negativo, bacterias +++ leucocitos $8-10$ por campo. $\mathrm{Hb}$ : de 12 g/.dl., glucemia de $133 \mathrm{mg} /$ dl creatinina de 1.1 colesterol de 292 $\mathrm{mg} / \mathrm{dl}$. y triglicéridos de 387

Son factores de riesgo para diabetes mellitus:

1.-___ La ocupación

2.-_ _L La edad de la paciente

3.-_ El género

4-__El El índice de masa corporal

5.-___El número de embarazos

6.-___ Las cifras de TA reportada

Son diagnósticos compatibles en el presente caso

7.-___ Diabetes mellitus autoinmune

8.-__ Diabetes mellitus tipo 2

9.-___ Hipertensión arterial controlada

$10 . \quad$ Infección de vías urinarias

11.__ Anemia

12._Enfermedad articular degenerativa.

13.__ Diabetes mellitus controlada

Son procedimientos diagnósticos útiles en el presente caso.

14._Glucemia en ayunas en forma mensual

15._Curva de tolerancia a la glucosa

16. Solicitar $\mathrm{HbA} 1 \mathrm{c}$

17._Depuración de creatinina en orina de 24 horas

18._Urocultivo

19._Perfil de lípidos en forma mensual

Son medidas terapéuticas apropiadas en el presente caso

20._Dieta de 1200 calorías

21._Glibeclamida 1 tab cada 12 horas

22._Metformin

23. Acarbosa al mediodía

24._Pravastatina 1 tableta en la noche

25. Bezafibrato 1 tableta en la mañana y otra en la noche

26._Diclofenac tabletas

27._Ampicilina oral

28. Caminata cada tercer día durante mínimo 30 minutos

Son medidas de seguimiento en el presente caso

29. Envío a Medicina Interna

30._Envío a Cardiología para toma de ECG

31._Revisión de fondo de ojo por el oftalmólogo

32. Cita mensual a Medicina Familiar

33._Glucemia en ayunas en forma mensual 